\title{
Cepheid and Long - Period Variables in Virgo Cluster Galaxies
}

\author{
M.J. Pierce ${ }^{1}$, R.D. McClure ${ }^{2}$, D.L. Welch ${ }^{3}$, R. Racine ${ }^{4}$ S. van den \\ Bergh $^{2}$
}

${ }^{1}$ Kitt Peak National Observatory, U.S.A., ${ }^{2}$ Dominion Astrophysical Observatory, National Research Council, Canada, ${ }^{3}$ McMaster University, Canada, ${ }^{4}$ Université de Montreal, Canada

\begin{abstract}
We are currently undertaking a ground-based imaging survey which attempts to discover and determine periods for variable stars in Virgo Cluster galaxies. Such a survey is now feasible thanks to the high resolution imaging (FWHM $\leq 0.50$ arcsec) routinely obtained with the High Resolution Camera on the Canada-France-Hawaii Telescope. The Virgo Cluster has long been considered a crucial "stepping-stone" in the extragalactic distance scale problem given that the cluster is at a "cosmologically interesting" distance and that there is little controversy in the relative distance between Virgo and more distant clusters, such as Coma. Consequently, much of the controversy regarding the extragalactic distance scale and the Hubble Constant can be eliminated with a determination of the Virgo Cluster distance. Some preliminary results and the prospects for establishing the distance to the Virgo Cluster using Cepheids and LPVs are discussed.
\end{abstract}

\section{The Extragalactic Distance Scale Problem}

It is generally believed that the historical discrepancy in estimates of the Hubble Constant prevents a definitive test of cosmological models. Much of the problem lies with the fact that the best understood distance indicators (e.g., Cepheid and RR Lyrae variables) are typically applicable over only modest distances, while the various secondary techniques (e.g., $\mathrm{D}_{n}-\sigma$, luminosity fluctuations, novae, planetary nebulae and globular cluster luminosity functions, supernovae, and the Tully-Fisher relations) are more uncertain and must rely on the primary methods for calibration. As a result, the uncertainties in the estimated distances to galaxies grow beyond the Local Group. However, the situation has recently improved now that quantitative prescriptions and cross-checks of the various secondary techniques have been developed. A comparison of these methods yields remarkably good agreement for the distance to the Virgo Cluster (e.g., Jacoby et al. 1992). Nevertheless, a more direct distance to the Virgo Cluster based on Cepheids would add considerable confidence to the current situation.

The Virgo Cluster is a key "stepping stone" in the extragalactic distance scale problem. Not only is it rich in galaxies over the full range of morphological types, 
but it contains enough systems that such relatively rare events, as supernovae, can be studied. Thus, Virgo appears to be an ideal environment for comparing and ultimately calibrating the secondary techniques. In addition, several of these methods can be used to estimate the relative distance between the Virgo and Coma clusters and hence sample the Hubble flow out to $\sim 7000 \mathrm{~km} \mathrm{sec}^{-1}$. The various estimates of the relative distance between these two clusters is also in very good agreement, so we can effectively "by-pass" any local peculiar velocities and estimate the Hubble Constant, provided that we can establish the distance to Virgo. It is generally believed that the detection and photometry of Cepheid variables in members of the Virgo Cluster will provide the most definitive estimate of the distance. Unfortunately, the current status of HST prevents this problem from being addressed for a few more years. In the meantime, it appears that the recent technical strides in high resolution imaging make a ground-based attempt at this problem feasible. In addition, we believe that Long-Period Variables (LPVs) may offer an interesting compliment/alternative to the Cepheids. An illustration of the promise of LPVs can be found in Pierce \& Crabtree (this volume). Here we report on some preliminary results of our attempts at discovering variables in Virgo Cluster spirals using HRCam at the CFHT.

\section{The Promise of High Resolution Imaging}

High resolution imaging produces significant gains in the problem of stellar photometry in external galaxies. Not only are the effects of crowding reduced with decreasing FWHM, but signal-to-noise increases linearly with decreasing FWHM in the background limited regime. Finally, "seeing" is a logarithmic quantity. That is, a 0.1 arcsec improvement in the FWHM at 1.2 arcsec is not nearly as significant as a similar improvement at a FWHM of 0.5 arcsec. Consequently, what might appear to be relatively modest gains in image quality at an already good site like Mauna Kea, are in fact quite significant. We also note that Cook, Aaronson, and Illingworth (1986) found Cepheids in M101 with the KPNO 4-m with FWHM 1.2 arcsec. Given that Virgo Cluster is at most a factor of 3 more distant, we can expect a similar chance of success if we can attain a FWHM $\sim 0.4$ arcsec. Such a goal is now within reach thanks to recent progress in ground-based imaging; in particular the success of the High Resolution Camera (HRCam) on the CFHT (see below). A similar argument for the potential gains from high resolution imaging of Virgo Cluster galaxies and some preliminary results can be found in Tanvir et al. (1991).

HRCam is essentially a "fast guider" which operates at frequencies up to 200 $\mathrm{Hz}$. It is capable of correcting for the "tip-tilt" component of atmospheric seeing by monitoring a nearby guide star. The characteristics of HRCam have been described by Racine \& McClure (1989) and McClure et al. (1989). This instrument has been demonstrated to produce significant gains in FWHM, as is evident from Figure 1.

The distribution of image FWHM with HRCam is seen to peak at FWHM $=0.55$ arcsec and appears to have a "wall" at $\sim 0.40$ arcsec. This lower limit to the FWHM is probably imposed by the optical aberrations of the CFHT primary and the optics within HRCam (e.g., Racine et al. 1991). Efforts are ongoing to push this limit down 
to $\sim 0.3$ arcsec in the future. In any event, we will demonstrate that the current performance of HRCam is sufficient to address the problem of obtaining photometry for the brighter stellar populations in Virgo Cluster galaxies.

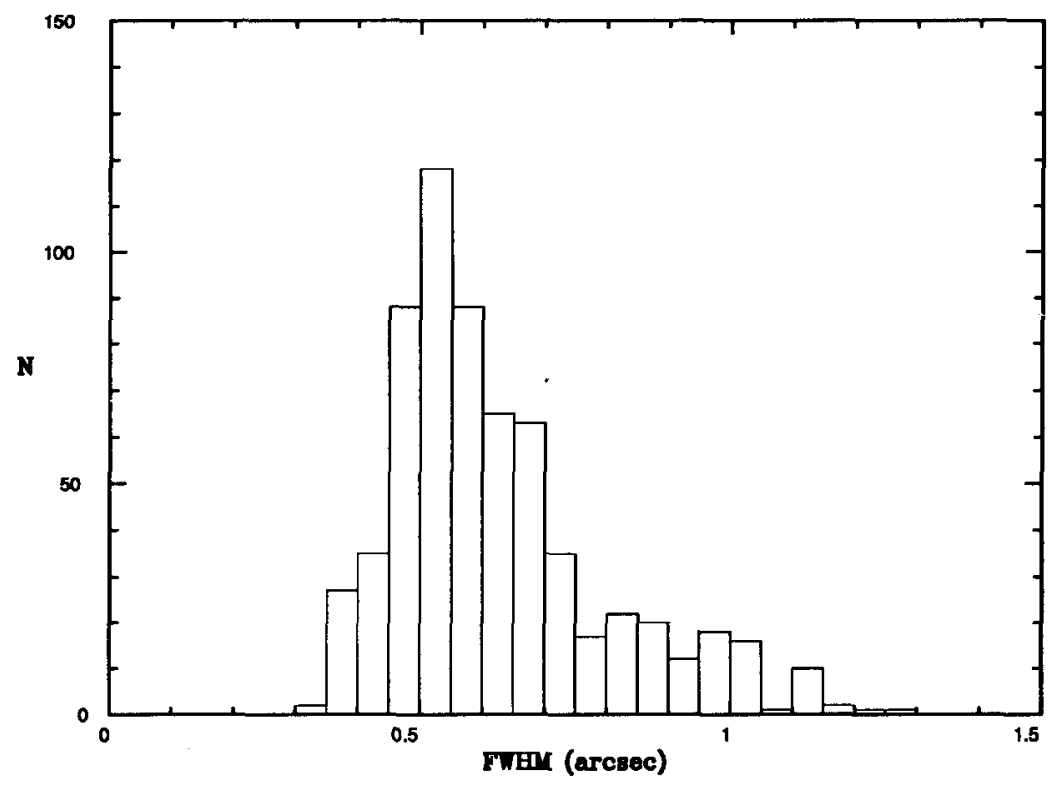

Figure 1 Histogram of measured FWHM with the CFHT with HRCam. The data are for every image (Jan. - Nov., 1991) with an integration longer than 10 sec.

The Virgo Cluster contains a considerable population of spiral galaxies from which we could choose our sample. Unfortunately, there is accumulating evidence for significant line-of-sight structure in the direction of the Cluster (e.g., Pierce and Tully 1988; Pierce 1991; Jacoby et al. 1992). This circumstance makes associating any small sample of spirals with the ellipticals, which might be presumed to lie in the inner core of the cluster, a somewhat dangerous approach. Nevertheless, it is the only way in which we can hope to establish the distance to the Virgo Cluster with Cepheids. To guard against this problem we have chosen two galaxies which are among the most likely to be in the inner core of the cluster. These are NGC 4571 and NGC 4321 . The first system is a relatively face-on spiral, of moderate luminosity, which has a very low radial velocity $\left(348 \mathrm{~km} \mathrm{sec}^{-1}\right)$. This implies it is within the core of the cluster, as any reasonable estimate of the Hubble Constant would place a foreground system so close that it would have been resolved into individual stars long ago. In fact, Virgo is the only region outside the Local Goup where galaxies have negative radial velocities, due to the large velocity dispersion in the cluster. NGC 4571 also shows strong evidence for being stripped of its outer envelope of $\mathrm{H} \mathrm{I}$ (e.g., van der Hulst et al. 1987). These two facts almost certainly place NGC 4571 in the core of the cluster. The second galaxy, NGC 4321 , is a relatively rare, luminous 
Sc I spiral, and has been argued to be a member of the cluster core on this basis (e.g., Sandage 1992). In addition, this system has produced a well observed type II supernova which allows a distance estimate via the "Baade-Wesselink", or expanding photosphere method (e.g., Schmidt, et al. 1992), and we can thus provide a direct test of this method.

\section{A Search for Variables in the Virgo Cluster with HRCam on the CFHT}

For our search program, we typically take 1 - 1.5 hours of integration in the R-band, breaking this into individual 15-min exposures to allow for cosmic ray discrimination and to give us some flexibility during conditions of variable seeing. The images are registered and combined with a clipping algorithum to produce a single image. To date, we have acquired 6 epochs with a FWHM $\leq 0.50$ arcsec for NGC 4571 and an additional epoch with FHWM $=0.55$ arcsec. We have only acquired 2 epochs for NGC 4321 (FWHM $~ 0.55$ arcsec), so we will discuss only NGC 4571 here. An example of our "plate material" is shown in Figure 2.

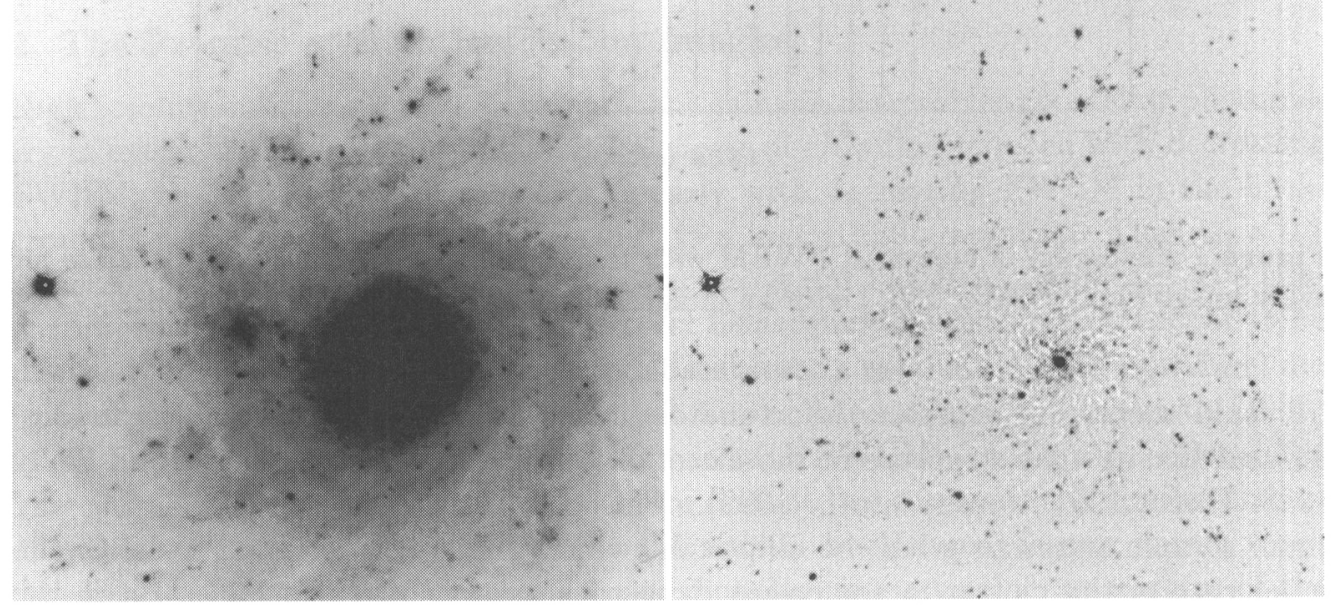

Figure 2 a) R-band image of NGC 4571 (1.5 hours, FWHM $=0.42 \operatorname{arcsec}$ ). b) The same image following the removal of the background light of the galaxy (see text). The individual stars are clearly resolved and range from $\mathrm{R} \sim 21$ for the brightest to $\mathrm{R} \sim 26$ for the faintest.

In the first panel we show one of our images (FWHM $=0.42$ arcsec). The resolution of the galaxy into stars is obvious, as is the rapidly varying background upon which they are found. In order to suppress the background and obtain stellar photometry we go through a few iterations of DAOPHOT, followed by smoothing. That is, we: 1) construct a PSF from the brightest isolated stars, 2) identify stars and subtract them, 3) smooth the subtracted image to suppress the residuals, and 4) subtract this "model" of the background from the original image. The process is repeated a few times, progressively decreasing the size of the smoothing kernel. The 
second panel illustrates the result of this procedure. The background light of the galaxy is suppressed, revealing numerous individual stars. In addition, the procedure reveals the presence of considerable dust complexes associated with the spiral structure of the galaxy. This image can be compared with that shown in Sandage and Bedke (1988) to give some idea of the improvement made possible with HRCam and the CFHT.



Figure 3 R-band luminosity function for stars in NGC 4571 from DAOPHOT. Note that incompleteness begins below $m_{R} \sim 24.0$ and that our limiting magnitude is about $m_{R} \sim 26$. The dashed lines indicate the expected $\mathrm{R}$ mag. at mean light for 50 day Cepheids, assuming distances of 15 and $22 \mathrm{Mpc}$ (see text).

Some preliminary results of our photometry of the resolved stellar population in NGC 4571, and a more extensive discussion of the feasibility of detecting of Cepheids, can be found in Pierce, McClure, \& Racine (1992). Figure 3 shows the R-band luminosity function of NGC 4571 taken from that paper. The two vertical lines indicate the expected $\mathrm{R}$ magnitude for 50 day Cepheids at mean light assuming Virgo distances of 15 and $22 \mathrm{Mpc}$. Since the luminosity function is empirical, we can estimate the completeness as $\sim 50 \%$ and $10 \%$ for the two distances by assuming a simple power law form. The brightest Cepheids ( $\mathrm{P} \geq 50$ days) are relatively rare and tend to have lower amplitudes, while those with $\mathrm{P} \leq 30$ days suffer from aliasing due to the lunar cycle. The shortest periods ( $\mathrm{P} \leq 20$ days) are simply too faint for us to detect. Consequently, we should be sensitive to only a narrow range in period and any aliasing problems should be minimal. With the 6 epochs acquired to date $\sim 40$ variable star candidates have been identified in NGC 4571 . We have 
used both blinking and the photometry to assess the reality of the variations. A few of the variables we have found are shown in Figure 4. The data span approximately 600 days. The first two epochs are from the 1991 season, while the remainder were obtained in 1992. The variation is clearly seen by comparing the apparent brightness of each of the identified stars with their neighbors. The variables we have identified have $24 \leq m_{R} \leq 25$ and amplitudes of $\sim 1 \mathrm{mag}$. The photometric errors at this level are $\sim 0.15$ mag. per epoch and this should provide adequate period determination with about 10 epochs.

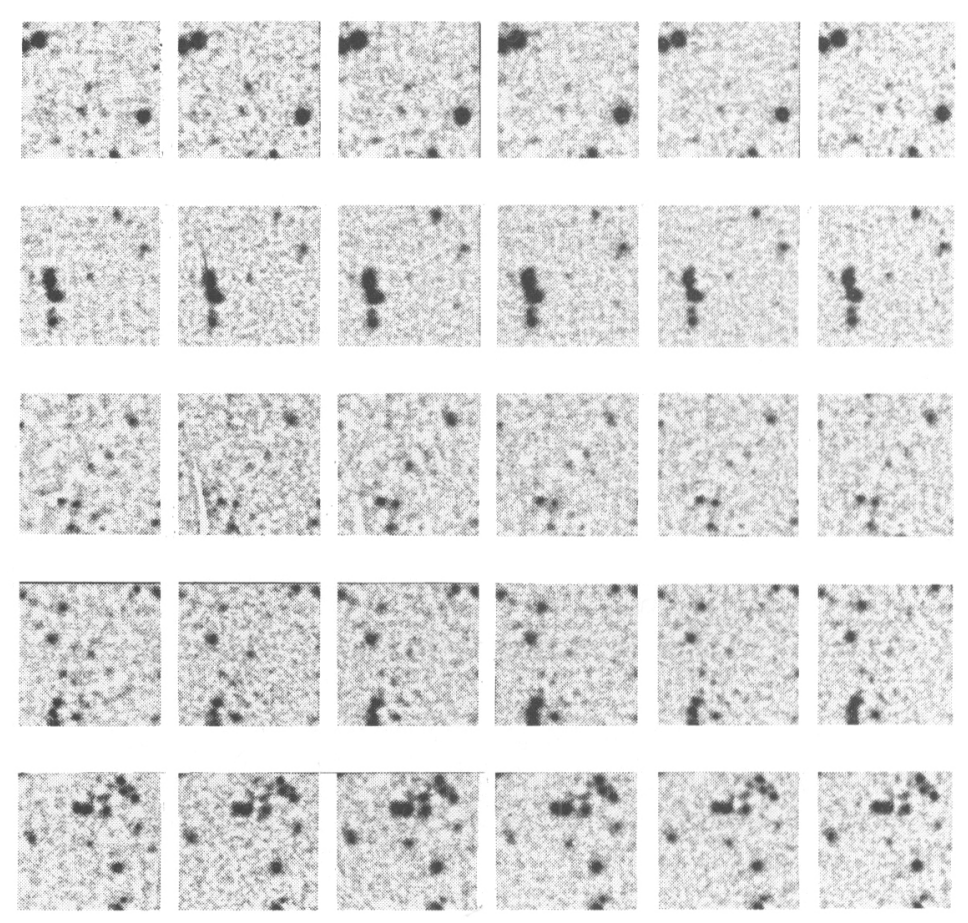

Figure 4 Subrasters centered around selected variables in NGC 4571 illustrating the six epochs acquired to date. The variations are made fairly obvious by the presence of nearby stars of comparable brightness. The background fluctuations due to the underlying population of $\mathrm{K}$ giants is also apparent. The non-uniformities in the background due to this incipient resolution and dust complexes are the limiting factor in our photometry.

Scaling the number of variables in the LMC by the relative luminosity of the galaxies, and accounting for our incompleteness, we should detect $\sim 3-8$ Cepheids in NGC 4571, depending on the actual distance. The crowding of stellar images should have no effect on the periods but will bias the mean light of the variables to brighter levels. On the other hand, crowding will result in decreased amplitudes and lower the probability of detecting the most contaminated variables. We plan to assess 
the result of these competing effects through simulations.

It is interesting to note that the variables in NGC 4571 are 5 magnitudes fainter than similar variables in members of the Local Group. This clearly demonstrates that significant gains are possible with high resolution imaging. With the small number of epochs in hand, we cannot yet determine periods. Nevertheless, we can still get some idea of the nature of these variables by looking at colors. Figure 5 shows a colormagnitude diagram for a selection of stars in NGC 4571 for which we have adequate V-band photometry.

The "plume" of blue supergiants near $V-R \sim-0.2$ is evident and suggests that the brightest blue stars suffer relatively little extinction. We might expect the same for any Cepheids we find since B main sequence stars are the progenitors. The brightest red supergiants are apparent near $R \sim 22$ with $V-R \sim 1.0$. They imply a distance modulus of $\sim 31$ mag., or $\sim 15 \mathrm{Mpc}$ (Pierce, McClure, \& Racine 1992). The majority of the variables $(\sim 80 \%)$ have $V-R$ colors of $\sim 1.0$, but a few have $V-R \sim 0.3$. The former are almost certainly LPVs, while we suspect the latter are Cepheids.

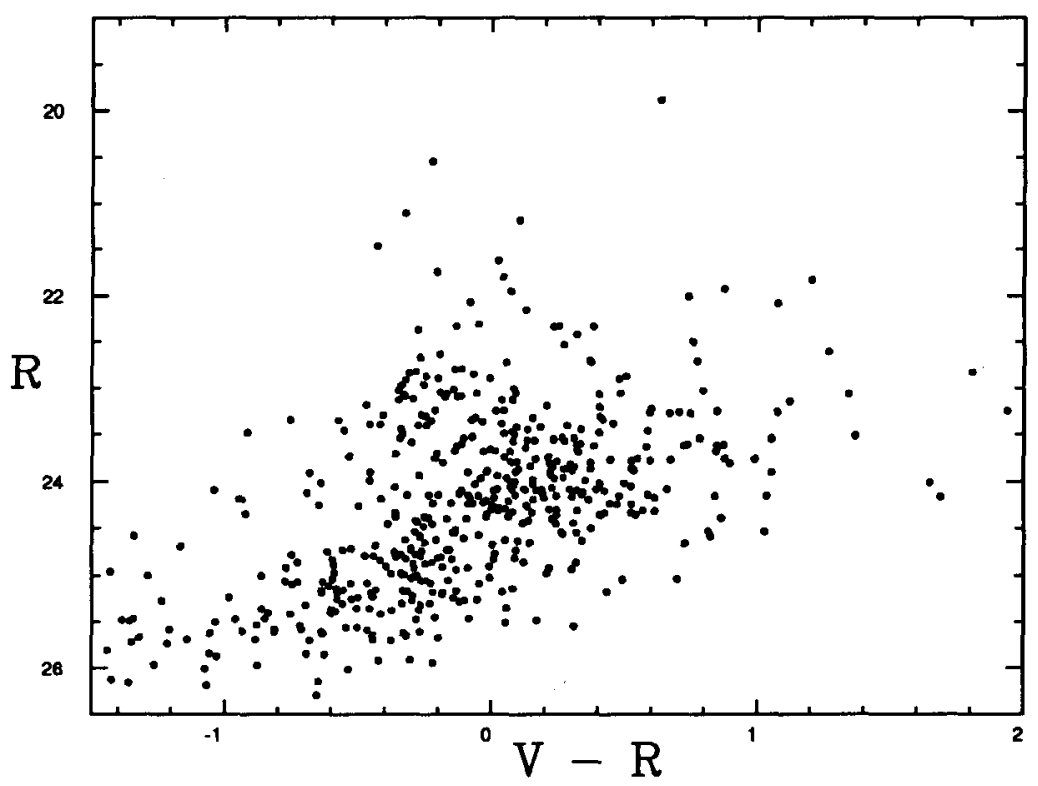

Figure $5 \mathrm{R}$ vs. $\mathrm{V}-\mathrm{R}$ color magnitude diagram for the resolved stars in NGC 4571. The "blue plume" of supergiants is obvious at $V-R \sim-0.2$, as well as the red supergiants at $R \sim 22$ and $V-R \sim 1.0$. The distribution of the fainter stars is due primarily to limitations in our V-band photometry.

LPVs offer an additional tool for estimating the distance to Virgo Cluster galaxies. A period-luminosity relation has been found for LPVs in the K-band with a dispersion comparable to that of the Cepheids (e.g., Wood, Bessell, \& Paltoglou 1985). 
However, the LPVs in Virgo are expected near $\mathrm{K} \sim 21$ and photometry will be extremely difficult. An alternative is to apply the optical $\mathrm{P}-\mathrm{L}$ relation found for these variables (Pierce \& Crabtree, this volume). By using a narrow band filter to isolate the continuum between the strong TiO found in these stars a random-phase $\mathrm{P}-\mathrm{L}$ relation with a dispersion of $\sim 0.25$ mag. has been found. There are no signs of any obvious systematics due to differences in metallicity. Given that LPVs are $\sim 5$ times more common than Cepheids of similar brightness means that we can expect to detect considerably more LPVs in distant galaxies than Cepheids. The same arguement also applies to the problem of the Galactic calibration as well. In fact, 10 LPVs are known in the Per OB1 association alone. So, it would appear that LPVs may turn out to be a useful alternative to Cepheids in estimating extragalactic distances.

\section{References:}

Cook, K., Aaonson, M., \& Illingworth, G. 1986, ApJ, 301, L45.

Jacoby, G., et al. 1992, PASP, 104, 598.

McClure, R. et al. 1989, PASP, 101, 1156.

Pierce, M. 1991, in Observational Tests of Cosmological Inflation, ed. T. Shanks, A.J. Banday, R.S. Ellis, C.S. Frenk, \& A. W. Wolfendale, NATO ASI Series C 348, p. 173.

Pierce, M., McClure, R., \& Racine, R. 1992, ApJ, 393, 523.

Racine, R., \& McClure, R. 1989, PASP, 101, 731.

Racine, R., Salmon, D., Cowley, D., \& Sovka, J., 1991, PASP, 103, 1020.

Sandage, A., \& Bedke, J. 1988, Atlas of Galaxies Useful for Measuring the Cosmological Distance Scale, NASA Scientific and Technical Information Division, (Washington: NASA)

Sandage, A. 1992, preprint

Schmidt, B., et al. 1992, preprint

Tanvir, N., et al. 1991, MNRAS, 253, 21P

van der Hulst, J., Skillman, E., Kennicutt, R., Bothun, G. 1987, AA, 177, 63.

Wood, P., Bessell, M., \& Paltoglou, R. 1985, ApJ, 290, 477.

\section{Questions}

J. Percy: In view of the fact that supergiant LPVs can be rather irregular, are you sure that you can use them reliably for distance determination? Will they be comparable in galaxies with perhaps different metallicities?

M. Pierce: The nice feature of the $P-L$ relation for $L P V s$ is that it is so flat that in some sense we don't even need accurate periods. We don't see any evidence of metallicity effects between the Galaxy and the LMC but it is something we are continuing to look into.

J. Matthews: Do the monthly gaps induced by the spacing of "dark time" really improve the aliasing situation? There could be Cepheids with periods as short as, say, $20 \mathrm{~d}$ for which you could not reliably identify a period! 
M. Pierce: Well, "improve" is certainly a poor choice of words. My point is only that the Cepheids as faint as, say, $20 \mathrm{~d}$ will be too faint to confuse with those at $40 \mathrm{~d}$, so we are likely to get periods with a minimal number of epochs.

N. Simon: What are the prospects for observing Cepheids with periods of about 15 days?

M. Pierce: I can't speak for HST, but the prospects using ground-based high resolution techniques appears slim. With FWHM $\sim 0.4$ arcsec they will be impossible to detect and more exotic adaptive optical systems will likely have such small fields as to make such a survey prohibitively expensive in terms of telescope time. 